\title{
\begin{tabular}{c} 
Case \\
Report \\
\hline
\end{tabular}
}

\section{Subarachnoid Hemorrhage due to Vertebral Artery Dissection after Lung Resection}

\author{
Sumiko Maeda, MD, PhD, Yoshinori Okada, MD, PhD, Akira Sakurada, MD, PhD, \\ Tetsu Sado, MD, PhD, Hisashi Oishi, MD, PhD, and Takashi Kondo, MD, PhD
}

\begin{abstract}
A 52-year-old man with massive hemoptysis underwent a left upper lobectomy. He suddenly reported a severe headache and lost consciousness on postoperative day 4. Computed tomography of the brain demonstrated a subarachnoid hemorrhage due to right vertebral artery dissection. Fortunately, he recovered well with intensive neurosurgical treatment. Vertebral artery dissection sometimes develops following systemic vascular disease, infection, and even minor neck trauma such as hyperextension or torsion of the neck. We should be aware that vertebral artery dissection may cause postoperative stroke and subarachnoid hemorrhage.
\end{abstract}

Keywords: lung resection, postoperative complication, stroke, subarachnoid hemorrhage, vertebral artery dissection

\section{Introduction}

Stroke is a rare but the most feared postoperative complication. The incidence of stroke depends on the type of surgery, $0.08 \%-0.7 \%$ in general surgery and $1.4 \%-9.7 \%$ in cardiovascular surgery. ${ }^{1)}$ Following lung resection for primary lung cancer in Japan, its incidence is $0.02 \%$, and it accounts for $1.7 \%$ of postoperative morbidity and $2.4 \%$ of total hospital deaths. ${ }^{2)}$ While the majority of postoperative strokes is embolic, hemorrhagic stroke and subarachnoid hemorrhage (SAH) are rarely reported. ${ }^{1)}$ The most frequent and well-known causes of non-traumatic SAH are rupture of a cerebral aneurysm and bleeding from a cerebral arteriovenous malformation. Vertebral artery (VA) dissection is a less frequent cause of $\mathrm{SAH}$; however, it is increasingly recognized as a cause of stroke and $\mathrm{SAH}^{3-5)}$

Department of Thoracic Surgery, Tohoku University Hospital, Sendai, Miyagi, Japan

Received: October 25, 2011; Accepted: December 26, 2011 Corresponding author: Sumiko Maeda, MD, PhD. Department of Thoracic Surgery, Tohoku University Hospital, 4-1 Seiryomachi, Aoba-ku, Sendai, Miyagi 980-8575, Japan

Email: sumaeda-ths@umin.ac.jp

(C)2012 The Editorial Committee of Annals of Thoracic and Cardiovascular Surgery. All rights reserved.
The case of a patient who underwent left upper lobectomy for massive hemoptysis because of an infected, chronic inflammatory granuloma is presented. SAH due to right VA dissection developed on postoperative day (POD) 4. The patient recovered successfully with intensive neurosurgical treatment. Postoperative SAH due to VA dissection is extremely rare, and to the best of our knowledge, this is the first report of such a case.

\section{Case Report}

A 52-year-old man was transferred to our hospital with acute respiratory failure due to massive hemoptysis. His past history included a median sternotomy for bilateral pneumothorax using surgical staplers at another hospital when he was 33 years old, but he had no hypertension or diabetes mellitus. He smoked one pack a day for 20 years. A day before transfer, he had massive hemoptysis and was taken to a hospital by ambulance. On admission to our hospital, he was under sedation and intubated with a tracheal tube with a blocker balloon. A chest X-ray demonstrated a loss of radiotransparency of the left lung (Fig. 1). His blood pressure was $92 / 57 \mathrm{~mm} \mathrm{Hg}$, pulse was 100 beats/min, and his oxygen saturation was $96 \%$ while he was on a mechanical ventilator with pure oxygen. The white-cell count was $13300 / \mathrm{mm}^{3}$, C-reactive protein 


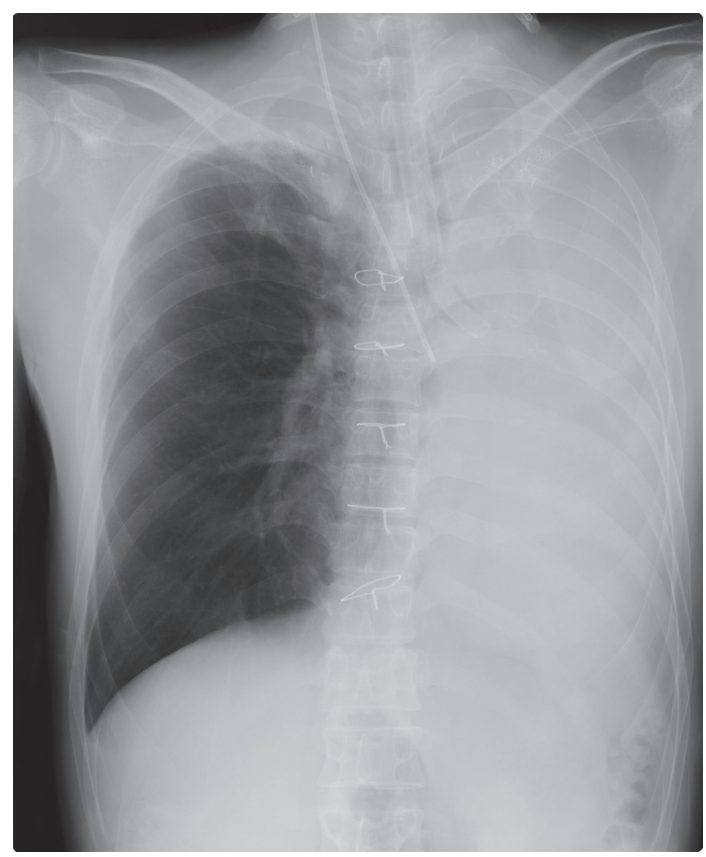

Fig. 1 A chest X-ray demonstrating total loss of radiotransparency of the left lung.

was $13.5 \mathrm{mg} / \mathrm{L}$, and the hemoglobin had decreased from $13.5 \mathrm{~g} / \mathrm{dL}$ to $9.8 \mathrm{~g} / \mathrm{dL}$. Initially, he underwent transcatheter arterial embolization, and 2 abnormal feeding vessels in the left upper lobe were embolized. On the second day, he underwent a left upper lobectomy. He was weaned from ventilatory support on POD2, but he was kept under moderate sedation because of his restless behavior due to delirium. He was discharged from the intensive care unit to a general ward on the morning of POD4. In the evening of POD4, when he was awake and alert, he suddenly reported a severe headache and lost consciousness. Computed tomography of the brain revealed diffuse hemorrhage in the basal cistern, and a 3-D reconstructed image indicated irregularly dilated intracranial right VA (Fig. 2); SAH due to right VA dissection was diagnosed. He underwent intensive neurosurgical treatment; transcatheter embolization of the right VA was performed on POD5, and relief of hydrocephalus was successfully achieved through a ventriculoperitoneal shunt. He recovered well with no motor impairment and was transferred to another hospital for rehabilitation on POD42. Hemoptysis never recurred after lung resection. Histological examination of the resected lung revealed pneumonia, bleeding, and abscess formation around chronic inflammatory granulation tissue. There was no evidence of malignancy or specific pathogens such as fungal or mycobacterial species.

\section{Discussion and Conclusion}

The VA has 4 anatomical segments: V1 is from the subclavian artery to the transverse foramen of C6; V2 is the segment ascending through the transverse foramen from C6-C2; V3 is from the transverse foramen of $\mathrm{C} 2$ and the foramen magnum; and V4 is the intracranial segment from the foramen magnum to the basilar artery. ${ }^{3)}$ The VA in V3 and V4 is vulnerable for anatomical reasons. The VA is mobile in V3 and relatively immobile in $\mathrm{V} 4$ at the point of penetrating the dura mater through the foramen magnum. ${ }^{6}$ In addition, the VA from the distal V3 to V4 has a thinner media and adventitia, with fewer elastic fibers than other segments. ${ }^{7)}$ Therefore, mechanical stress on the vessel wall in these segments may cause an intimal tear and intramural hemorrhage. An intimal tear may sometimes lead to a transmural rupture in V4.

VA dissection generally causes severe neck pain or an occipital headache on the affected side. The further clinical presentation may differ depending on the morphology of the dissection at angiography, the steno-occlusive type or the aneurysmal type. The steno-occlusive type is likely associated with infarctions in the area of the posterior circulation such as the lateral medulla, the cerebellum, and the spinal cord. ${ }^{4)}$ In contrast, the aneurysmal type, which is mostly located in V4, is likely to cause SAH. ${ }^{3)}$ Sometimes, VA dissection causes only pain in the neck without neurological impairments and heals spontaneously.

The pathogenesis of VA dissection is not fully understood. Underlying vascular disorders such as fibromuscular dysplasia and cystic medial necrosis, infections, trauma, hypertension, and oral contraceptives are reportedly associated with VA dissection. ${ }^{3,8)}$ The most commonly encountered factor is a wide variety of trauma to the head and neck. ${ }^{4)}$ Many reports have followed apparently trivial traumatic events, including performing yoga exercise, chiropractic manipulation, visiting a hairdresser, a fall during intoxication with alcohol, and even undergoing gastrofiberscopic examination under general anesthesia. ${ }^{4,910)}$ These procedures include hyperextension or torsion of the neck, which produces mechanical stress on the VA wall.

There are several possibilities as to when the present patient developed the VA dissection. He might have had an impending lesion before the present illness. Alternately, he might have developed the VA dissection at some time during his hospitalization, but symptoms such as neck pain or occipital headache were masked until the 


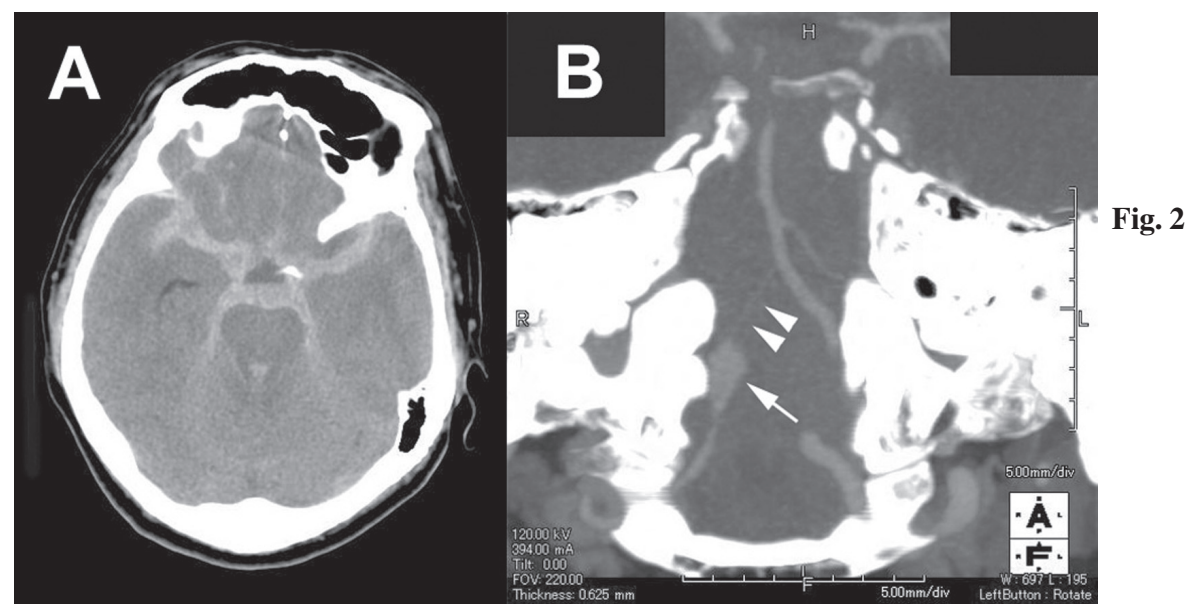

A: Computed tomography of the brain demonstrating diffuse hemorrhage in the basal cistern.

B: Three-dimensional reconstructed CT indicating an aneurysm of the right vertebral artery (arrow), followed by an irregularly narrow segment to the basilar artery (arrowheads), probably due to vasospasm. evening of POD4 because he was mostly intubated and sedated. In regard to possible reasons for his VA dissection, one is pulmonary infection. ${ }^{8)}$ Histological findings demonstrated pneumonia and abscess formation in the resected lung. The other is hyperextension or torsion of his neck. He was intubated or reintubated 3 times in total, and he was intermittently restless with intensive movements due to delirium even though he was sedated during his ICU stay.

When SAH and irregularly dilated intracranial VA were found in the CT images of the brain, VA dissection would be a highly probable cause of SAH. The 3-D reconstruction images of VA may not necessarily show an intimal flap. Atherosclerotic fusiform aneurysm and infected aneurysm would be considered as differential diagnosis based on the morphology of dilated vertebral artery; however, these conditions less likely cause SAH. A four-vessel study is the gold standard for diagnosing cerebrovascular disease. However, a 3-D reconstructed CT of the brain with contrast media was less invasive and useful to diagnose VA dissection in the present case. Ruptured VA dissections are unstable and easily rebleed. Overall, $70 \%$ of rebleeding occurs within a day after the initial SAH, with a very high mortality. ${ }^{11)}$ Therefore, an immediate diagnosis of ruptured VA is necessary for early intervention and a better prognosis.

In conclusion, it is important to be aware of VA dissection as a possible cause of postoperative stroke and subarachnoid hemorrhage, even though it is extremely rare.

\section{Disclosure Statement}

Sumiko Maeda and co-authors have no conflicts of interest.

\section{References}

1) Selim M. Perioperative stroke. N Engl J Med 2007; 356: 706-13.

2) Sakata R, Fujii Y, Kuwano H. Thoracic and cardiovascular surgery in Japan during 2008: annual report by The Japanese Association for Thoracic Surgery. Gen Thorac Cardiovasc Surg 2010; 58: 356-83.

3) Shin JH, Suh DC, Choi CG, et al. Vertebral artery dissection: spectrum of imaging findings with emphasis on angiography and correlation with clinical presentation. Radiographics 2000; 20: 1687-96.

4) McCrory P. Vertebral artery dissection causing stroke in sport. J Clin Neurosci 2000; 7: 298-300.

5) Zhao WY, Krings T, Alvarez H, et al. Management of spontaneous haemorrhagic intracranial vertebrobasilar dissection: review of 21 consecutive cases. Acta Neurochir (Wien) 2007; 149: 585-96.

6) Pollanen MS, Deck JH, Blenkinsop B. Injury of the tunica media in fatal rupture of the vertebral artery. Am J Forensic Med Pathol 1996; 17: 197-201.

7) Wilkinson IM. The vertebral artery. Extracranial and intracranial structure. Arch Neurol 1972; 27: 392-6.

8) Guillon B, Berthet K, Benslamia L, et al. Infection and the risk of spontaneous cervical artery dissection: a case-control study. Stroke 2003; 34: e79-81.

9) Weintraub MI. Beauty parlor stroke syndrome: Report of five cases. JAMA 1993; 269: 2085-6.

10) Testai FD, Gorelick PB. An unusual cause of vertebral artery dissection: esophagogastroduodenoscopy. Stroke Res Treat 2010: 915484.

11) Mizutani T, Aruga T, Kirino T, et al. Recurrent subarachnoid hemorrhage from untreated ruptured vertebrobasilar dissecting aneurysms. Neurosurgery 1995; 36: $905-11$. 